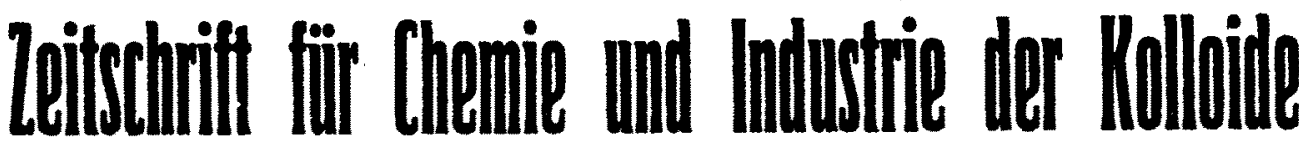 \\ Technische und wissenschaftliche Rundschau
}

für alle Industrien, welche mit anorganischen und organischen Kolloiden arbeiten.

............ Herausgegeben von Dr. Rudolf Ditmar .............

Inhaber des technisch-wissenschaftlichen Laboratoriums für die Oummi- und Leim-Industrie in Oraz.

Erscheint monatlieh!

Verlag von STEINKOPPF \& SPRINOER

Dresden-A. 21, Dombin̈thstrafe 40 .

Preis jhrlich M. 12.-

Die Redaktion ersucht höflichst alle Herren Autoren, welche über Kolloide arbeiten und ihre Forschungen in der Kolloidzeitschrift referiert haben möchten, dieselben zwecks möglichst pünktlichen Referates direkt an die Redaktion dieser Zeitschrift zu senden.

Die Redaktion hat sich im AnschluB an die Deutsche Chemische Oesellschaft entschlossen, eine allgemeine gleiche und konforme Rechtschreibung in dieser Zeitschrift einzuführen. Sie hält sich in Zukunft an das vom Verein Deutscher Ingenieure herausgegebene Büchlein: Rechtschreibung der naturwissenschaftlichen und technischen Fremdwörter" von Dr. Hubert Jansen und ersucht auch alle Mitarbeiter sich an diese Rechtschreibung zı halten.

Dr. R. Ditmar.

\section{Zur Kenntnis der Stabilität kolloidaler Lösungen.}

Von Dr. Jean Billitzer.

Unter diesem Titel veröffentlichte Herr Th. Svedberg in Nr. 6 dieser Zeitschrift interessante Beobachtungen, zu denen ich folgendes hinzufügen möchte.

Nach allem, was wir heute wissen, können die allgemeinen physikalischen Prinzipien für die Stabilität kolloidaler Lösungen in alkoholischen, allgemein organischen, wie überhaupt in beliebigen flüssigen Medien kaum andere sein wie in Wasser. Ihrer Natur, eine Art feinster Suspension vorzustellen entsprechend, sind die Bedingungen für die Stabilität kolloidaler Lösungen verschiedene, wenn man es in ihnen mit Teilchen zu tun hat, welche in elektrischem Gegensatze zum Medium stehen oder wenn dies nicht der Fall ist.

Ist ein elektrischer Gegensatz nicht vorhanden, so sind die Bedingungen der Stabilität:

a) Die schwebenden Teilchen besitzen dasselbe spezifische Gewicht wie das Medium.

Dieser Spezialfall, bei welchem auch grobe Suspensionen stabil wären, dürfte einen seltenen Ausnahmefall vorstellen. Die Stabilität wird ferner an bestimmte. Temperaturintervalle gebunden sein, es wäre denn, daß Medium und suspendierte Teilchen gerade gleichen Wärmeausdehnungskoeffizienten besitzen.

b) Besitzen die schwebenden Teilchen ein vom Medium verschiedenes spezifisches Gewicht, so werden sie den Gravitationskräften solange folgen können und also ausflocken, solange nicht die bei der Ausflockung zu überwindenden Reibungskräfte den Gravitationskräften gleich. kommen. Je größer die Oberfläche der suspendierten Substanz, also je feiner ihre Verteilung, je kleiner die schwebenden Teilchen sind, desto früher wird dieses dynamische Gleichgewicht erreicht, je geringer der. Unterschied der spezifischen Gewichte, je größer der Reibungskoeffizient, desto günstiger liegen die Bedingungen für die Stabilität. Mit der Aenderung dieser Größen durch Temperatur, Druck etc. ändert sich auch die Stabilität.

In diesen Fällen läBt sich bei Kenntnis der genannten physikalischen GröBen die Teilchengröße berechnen, bei welcher die Suspension resp. das Kolloid stabil werden.

Stehen hingegen die Teilchen in elektrischem Gegensatze zum Medium, so wird ihre Stabilitätsgrenze früher erreicht, weil die elektrostatischen ÁbstoBungskräfte, welche nun Teilchen auf Teilchen ausübt, der Koagulation entgegenwirken. Da wir die GröBe dieser Kraftwirkungen nicht berechnen können, vermögen wir nur qualitativ eine Erhöhung der Stabilität vorauszusagen, ohne sie quantitativ berechnen zu können. Zugleich mit Erlangung des elektrischen Gegensatzes erlangt das Kolloid aber auch die Eigentümlichkeit durch Elektrolytzusätze aus. gefällt werden zu können, weil nun die Konden. sationskernwirkung ungleichnamiger Ionen wirksam zu werden beginnt, wie ich es des Näheren 
in meiner Theorie der kolloidalen Lösungen ausgeführt habe 1 ).

Ueber die Erhöhung der Stabilität durch Eintritt des elektrischen Gegensatzes läßt sich aber folgendes sagen:

Sie wird cet par. umso größer sein, je größer der elektrische Gegensatz ist. Da elektrische Gegensätze resp. bei unbestimmter und geringer Ionenkonzentration Zufälligkeiten und Schwankungen unterworfen sind und da die lonenbildung cet. par. leichter auftritt, je größer die Dielektrizitätskonstante des Mediums oder lösungsmittels ist, wird die Stabilität im allgemeinen, sofern sje durch den elektrischen Gegensatz bedingt oder mitbedingt ist, um so eher in verschiedenen Löstungsmitteln erreicht werden können, je größer deren D. K. ist. Allein maßgebend ist die D. K. indessen durchaus nicht. Denn außer den sub $b$ genannten Momenten, welche ebenso wirksam sind wie bei ungeladenen Kolloiden, kann der elektrische Gegensatz (der durch elektromotorische Kräfte oder durch den verschiedenen Teilungskoeffizienten der lonen zwischen Lösungsmittel und schwebendem Körper bestimmt wird) nur dann konstante und gleiche Stabilität herbeiführen, wenn er selbst konstant ist. Bedingung für die Konstanz der Potentialdifferenz ist aber ein bestimmter elektromotorischer Vorgang oder bestimmter lonenverteilung eine konstante Konzentration bestimmter Ionengattungen bei konstanter Temperatur und bei sehr kleinen Ionen-Konzentrationen wohl auch eine genügend große Ionenbildungsgeschwindigkeit, und diese Bedingungen sind zwar häufiger in Medien großer D. K. erfüllt als in anderen, doch ist eine große D. K. für sich allein nicht mafgebend.

Gerade bei organischen Lösungsmitteln liegen nun die Bedingungen für die Konstanz in der Regel recht ungünstig. In Alkoholen ist z. B. die lonenkonzentration von Haus aus wohl bestimmt, aber durch Säurebildung unter Mitwirkung des Luftsauerstoffes (bei LuftabschluB des Sauerstoffes der an den Glaswandungen festgehaltenen Luft, des gelösten Sauerstoffes etc.) ändert sich dieselbe schnell. Es bilden sich bekanntlich superoxydartige Körper, von denen nur eine Spur genügt, um die Potentialdifferenzen von Sauerstoffelektroden, wie sie bei Platin vorliegen, ganz wesentlich zu verändern usf.

Deshalb kommt es wohl, daß Stabilitäten von kolloidalen Lösungen in solchen Medien wer $\mathrm{zu}$ reproduzieren sind und zufälligen

1) Cf. der Ztschr. f. phys. Chem. 51, 130 (1905).
Charakter tragen, weil sich eben die Zusammen. setzung des Mediums in unkontrollierbarer Weise ändert. So habe ich vor mehreren Jahren oft Platin in Aethylalkohol zerstäubt und erhielt wie Svedberg (l. c.) meist wenig stabile, manchmal aber ziemlich stabile Alkosole. In letzteren wanderte das Platin zur Kathode, was ich auf die Wirkung der Autoxydationsprodukte des Alkohols zurückzuführen geneigt war. Svedberg erhielt hingegen in Alkohol anscheinend nur instabile Sole. Auch in Chloroform ist nach seinen Angaben Platinsol instabil. Ich habe aber ill Chloroform vor mehreren Jahren Platin zerstäubt und Platinsole erhalten, dic stabil genug waren, um zu mehrstündigen, zeitweilig mehrtägigen Versuchen verwendet zu werden. Auch in Chloroform wandert das Platin zur Kathode, ich brachte dieses Sol in eine U-Röhre, schichtete in einem Schenkel schwach alkalisches Platinhydrosol (in welchem Platin zur Anode wandert) darüber und verband das Hydrosol mit dem negativen, das Chloroformsol mit dem positiven Pole, von beiden Seiten wanderte nun das Platin gegen die Trennungsfläche Chloroform/Wasser und fiel dort, wie ich es erwartet hatte, aus.

DaB die Stabilität auBer von dem mehr oder minder zufälligen Zustande des Lösungsmittels wegen ihres Einflusses auf die Potentialdifferenz auch von der Temperatur und der Natur des elektromotorischen Vorganges, mithin der Natur des zerteilten Stoffes abhängig ist, erscheint ganz selbstverständlich und ist von mir bereits (1. c.) betont worden. $\mathrm{DaB}$ die Koagulation eine um so schnellere ist, je höher die Temperatur bei überschrittener Stabilität ist, klingt ebenso selbstverständlich.

Svedberg findet, daß die Perrin'sche Regel, die Stabilität kolloidaler Lösungen hänge mit der D. K. zusammen, nicht bestätigt wird, und dies ist durchaus nicht überraschend, denn die Ionisierungskraft des Lösungsmittels bildet, wie wir gesehen haben, nur einen der vielen Faktoren, welche mit der Ionisierung die Aeußerung elektromotorischer Kräfte und mit ihnen die Herbeiführung elektrischer Gegensätze oft begünstigen.

Auch die Burton'sche Regel wird nicht bestätigt und würde sie es, so müßte das mindestens ebenso wunderbar sein, wie die Erscheinungen, die sie erklären will, sie wäre daher mindestens ebenso erklärungsbedürftig wie jene.

Einen Ueberblick über die Erscheinungen kann man erst dann gewinnen, wenn man zu- 
vor der Natur der elektromotorischen Kräfte nachgeht und auch die sub $a$ und $b$ genannten Faktoren berücksichtigt. Ergibt es sich dann, $\mathrm{daB}$ die heutige Theorie unzureichend ist, so muß sie ergänzt werden oder abgeändert, wenn sie zu Widersprüchen führt. Heute liegt aber weder das Bedürfnis zu einer Ergãnzung noch zu einer Abänderung, wohl aber das nach einer genaueren Kenntnis der Erscheinungen selbst vor.

\section{Ueber das Verhalten von Farbstoffen gegen die Hydrosole der Silberhalogenide.}

Von Dr. Lüppo-Cramer.

(Mitteilungen aus dem wissenschaftlichen Laboratorium der Trockenplattenfabrik

Dr. C. Schleu B ner, Akt.-Ges., Frankfurt a. M.)

Als erste Bedingung für die optische Sensibilisierung des Bromsilbers durch Farbstoffe wurde bereits im Jahre 1884 von Eder ${ }^{1)}$ die Adsorption des Farbstoffes durch das Bromsilberkorn aufgestellt. Später hat dann auch von $\mathrm{Hübl}^{2}$ ) hierüber eingehende Untersuchungen veranstaltet, welche die Eder'schen Befunde bestätigen. Der Verfasser ${ }^{3}$ ) fand ferner bei Versuchen über angefärbtes Bronssilber und andere unlösliche Körper die Gesetzmäßigkeit, daB Salze mit solchen Anionen, die denen des angefärbten Körpers gleich sind, diesem den Farbstoff teilweise entziehen. Interessante Untersuchungen veröffentlichte auch neuerdings Karl Kieser in seiner Inaugural-Dissertation $*$ Beiträge zur Chemie der optischen Sensibilisation von Silbersalzen « Freiburg i. Br. 1904. Auf die Bedeutung der KorngröBe des Bromsilbers für die optische Sensibilisierung wies ich mehrfach bei Versuchen über das Lippmann'sche Farbenverfahren ${ }^{4}$ ) hin, bei welchem, wie wir in einem früheren Artikel s) sahen, das kolloidale Bromsilber vorliegt, das bei seiner außerordentlich großen Oberfläche in bedeutend höherem Grade der Sensibilisierung zugänglich ist, der $*$ chemischen " sowohl wie der optischen.

In neuester Zeit ist namentlich durch die Untersuchungen von Zsigmondy ${ }^{6}$ ) und von Wilhelm Biltz ${ }^{7}$ ) die vollständige Nachbildung des Färbevorganges mit rein anorganischem Material gelungen, womit die Frage nach der S. 152.

1) Eders Handb. d. Photogr., III. Bd., 5. Aufl.,

2) Eders Jahrb. f. 1891, S. 189.

3) Archiv f, wissensch. Photogr. II., 1901, S. 277.

4) Eders Jahrb. f. 1901, S. 24; 1902 , S. 59.

5) Diese Ztschr. Bd. 1., S. 165.

6) Zsigmondy, Zur Erkentnis der Kolloide Jena 1905.

7) Nachrichten der K. Gesellsch. d. Wissensch. zu Göttingen, Mathein.-physik. KI. 1904 Heft 1 und 1905 Heft 1; ferner auch Ber. der deutsch. chem. Gesellschaft 1904, 272 und 1905, 521 u. 522.
Natur des Anfärbeprozesses zu einem gewissen Abschlusse gelangt und zugunsten der Adsorptionstheorie entschieden zu sein scheint. Zsigmondy beobachtete zuerst, daB kolloidales Gold gebeizte Wolle anfärbt und mit frisch gefällter Tonerde einen rot gefärbten Lack bildet, und Biltz wies nach, daß einerseits sich die thierische und pflanzliche Faser (Seide, Wolle, Baumwolle) mit anorganischen Kolloiden (Selen, Gold, Vanadinoxyden, Molybdänblau, Arsen - und Antimonsulfid etc.) anfärben läBt, andererseits aber auch die Faser durch ein anorganisches Hydrogel $\left(\mathrm{Al}_{2} \mathrm{O}_{3}, \mathrm{Zr} \mathrm{O}, \mathrm{Fe}_{2} \mathrm{O}_{3}, \mathrm{Sn} \mathrm{O} \mathrm{O}_{2}\right)$ ersetzt werden kann, ohne daB der quantitative Verlauf der Adsorption von Farbstoffen geändert wird. Von Bedeutung für die im folgenden zu beschreibenden Versuche ist hier noch der von Krafft und Preuner $^{8}$ ) gelieferte Nachweis zu erwähnen, daB die substantiven Farbstoffe, speziell die Benzidinfarbstoffe, sich in Wasser nicht als Elektrolyte, sondern als Kolloide lösen. Bezüglich der Farbstoffe der Eosinreihe ist wohl jedem Photochemiker, der sich mit der Reinigung z. B. von Erythrosin beschäftigt hat, die Erscheinung bekannt, daß sich das z.B. mit verdünnter Schwefelsäure ausgefällte Erythrosin (Tetrajodfluorescëinnatrium) zwar zuerst klar abfiltrieren läBt, nach einigen Waschungen dann aber intensiv gefärbt durchs Filter geht. In diesem Falle ist die Säure des Erythrosins in kolloidale Lösung getreten.

Untersuchungen über die Wirkung von Farbstoffen auf die Hydrosole der Silberhalogenide gaben mir einige interessante Resultate. Das Hydrosol des Bromsilbers wurde nach dem Prinzip von Lottermoser in sehr verdünnter Lösung für jede Versuchsserie frisch hergestellt. Die Farbstoffe verdanke ich der

8) Nach W. Biltz, Nachr. d. K. Ges. d. Wiss. z. Göttingen, Mathem.-physik. KI. 1904 Heft 1, S. 12, Ber. d. deutsch. chem. Oes. 1904, S. 1773. 